\title{
XBPIs Acts as a Tumor Suppressor to Inhibit the EMT Process and Metastasis of Papillary Thyroid
} Cancer

\author{
Wen Yang ${ }^{1, *}$ \\ Xia $X u^{2, *}$ \\ Ming $X u^{\prime}$ \\ Jun Zhou iD \\ Zihan $\mathrm{Xi}^{\mathrm{I}}$ \\ Hui Guo' \\ Jie Ming \\ Tao Huang' \\ 'Department of Breast and Thyroid \\ Surgery, Union Hospital, Tongji Medical \\ College, Huazhong University of Science \\ and Technology, Wuhan, People's \\ Republic of China; ${ }^{2}$ Department of \\ Pathology, Union Hospital, Tongji Medical \\ College, Huazhong University of Science \\ and Technology, Wuhan, People's \\ Republic of China
}

*These authors contributed equally to this work
This article was published in the following Dove Press journal: OncoTargets and Therapy

Purpose: Papillary thyroid cancer (PTC) patients could obtain poor prognosis if they have lymph node metastasis, identification of informative and robust biomarkers for predicting cervical lymph node metastasis is critical for improving clinical decision-making and patient prognosis.

Materials and Methods: In this study, we analyzed the expression of $\mathrm{X}$ box binding protein 1 spliced-form (XBP1s) in 41 PTC tissue samples and extended our findings using public databases, then we investigated how XBP1s' contributed to PTC progression in vitro. Results: We found that XBP1s' expression was lower in PTC patients with cervical lymph node metastasis than non-metastasis patients by immunohistochemical analysis. With publicly accessible dataset, we showed that the XBP1 transcription was significantly decreased in thyroid cancer (TC) tissues with lymph node metastasis as compared to that without lymph node metastasis. Moreover, we also found that XBP1 expression was significantly correlated with patients' gender, T classification, lymph node metastasis and PTC stages, and low XBP1 expression was associated with poor diseases free survival (DFS). In vitro, XBP1s overexpression could inhibit the invasion, migration, and wound healing capacity of PTC cells. Mechanistically, overexpression of XBP1s could enhance the expression of classical epithelial-mesenchymal transition (EMT) markers such as ZO-1 and E-cadherins, and downregulated $\mathrm{N}$-cadherin in BCPAP cells.

Conclusion: These findings suggest that XBP1s is a prognostic maker for thyroid carcinoma patients, and sustaining XBP1s expression might be a new strategy to control PTC progression.

Keywords: XBP1s, lymph node metastasis, papillary thyroid cancer, EMT

\section{Introduction}

Papillary thyroid cancer (PTC) consists of around $80 \%$ of thyroid cancers. ${ }^{1}$.The prognosis of most PTC patients is favorable with the standard treatments. ${ }^{2}$ However, there is a small subset of PTC patients with lymph node metastasis has poor prognosis. ${ }^{3}$ Thus, identification of informative and robust biomarkers for predicting cervical lymph node metastasis is critical for improving clinical decision-making and patient prognosis.

$\mathrm{X}$ box binding protein 1 (XBP1) plays a critical role in regulating the unfolded protein response (UPR). UPR is an evolutionarily conserved signaling pathway regulated by endoplasmic reticulum (ER) stress, which occurs when unfolded and misfolded proteins accumulate in the lumen of the ER. ${ }^{4,5}$ Inositol-requiring enzyme 1 (IRE1) is an important
Correspondence: Tao Huang; Jie Ming Department of Breast and Thyroid Surgery, Union Hospital, Tongji Medical College, Huazhong University of Science and Technology, Jiefang Road No. 1277, Wuhan, Hubei Province, People's Republic of China

Tel $+86 / 3807 \mid I 2766 ;+8613905519049$

Fax $+8627-85726833$

Email huangtaowh@163.com;

mingjiewh@I26.com 
RNase involved in ER stress to splice the XBP1 mRNA to generate XBP1 spliced-form (XBP1s), which serves as an active transcription factor. Previous studies have shown that $\mathrm{XBP} 1 \mathrm{~s}$ is a novel regulator for carcinogenesis and tumor metastasis in multiple types of cancer, but the detail mechanisms are quite different in various tumors. ${ }^{6-8}$ For instance, XBP1s directly activates c-MYC and regulates oncogenic c-MYC signaling in prostate cancer (PCa) cell lines, high expression of XBP1s is associated with poor survival in $\mathrm{PCa}$ patients. ${ }^{9}$ In triple-negative breast cancer, XBP1s interacts with HIF-1 $\alpha$ to cooperatively activate HIF-1 targeted genes. ${ }^{10}$ Intriguingly, it has been shown that intestinal stem cell numbers were increased in mice lacking XBP1, and loss of XBP1 leads to oncogenic events activation through IRE1 $\alpha$ and STAT3 signaling. ${ }^{11}$ Furthermore, it is uncovered that XBP1s exerts antiproliferative function via the interaction with PERKeIF2 $\alpha$ in colorectal cancer cells. ${ }^{12}$

Although XBP1s has been identified to play multiple roles in tumor progression, the function of XBP1s in thyroid cancer has not been well characterized so far. In this study, we analyzed XBP1s' expression in 41 PTC tissue samples and extended our findings using public databases, then we investigated how XBP1s' contributed to PTC progression in vitro.

\section{Materials and Methods}

\section{Patient Samples}

A total of 41 patients with PTC were included in this study. All patients underwent thyroidectomy between March 2015 and October 2018. We obtained the formalin-fixed, paraffinembedded (FFPE) tissue blocks from the Pathology Department of Union Hospital (Wuhan, China). The patients did not receive any chemotherapy or radiotherapy before surgery. Written informed consent was obtained from every patient whose clinical specimens were used for the current research. The study was approved by the Ethics Committee of Tongji Medical College, Huazhong University of Science and Technology, China. We confirm that all tissue samples were used in accordance with the Declaration of Helsinki.

\section{Immunohistochemistry (IHC)}

Immunohistochemical staining was carried out as described previously. ${ }^{9}$ Slides were incubated with anti-XBP1s (1:100 dilution; CST, \#83418). All the IHC results were evaluated by two experienced pathologists that are blind to the diagnosis and independently. To assess the expression of XBP1s, the procedure was as following: 1 , accessing the tissue staining score according to the staining intensity $(0$, negative; +1 , weak positive; +2 , moderate positive; +3 , strong positive); 2 , calculating the percentage of positive tumor cells; 3 , calculating the staining index [SI $=($ intensity score in 1$) \times($ positive percentage)]. $\mathrm{SI} \geq 1.05$, the optimal critical value determined by ROC curve, was classified as high expression.

\section{Data Acquisition of Database}

The expression of XBP1 and correlation with the clinicopathological characteristics of thyroid cancer patients were analyzed using two visual analysis websites, GEPIA (http://gepia.cancer-pku.cn/) and UALCAN (http://ualcan. path.uab.edu). The TC patients raw data including the clinical information and RNA sequencing were downloaded from The Cancer Genome Atlas (TCGA) repository website (http://firebrowse.org/). We analyzed all samples with lymph node metastasis information $(n=451)$.

\section{Cell Culture}

PTC cell lines, BCPAP, KTC-1 and TPC-1 were purchased from the American Type Culture Collection (ATCC). Cells were cultured in RPMI 1640 with $10 \%$ fetal bovine serum (FBS) at $37^{\circ} \mathrm{C}$ in $5 \% \mathrm{CO}_{2}$.

\section{Cell Transduction}

BCPAP, KTC-1 and TPC-1 cells were transduced with lentivirus (overexpression of XBP1s (Obio, Shanghai, China) and control) using co-transfection reagent $\mathrm{P}$ (Genechem, Shanghai, China). Cells were cultured with $3 \mu \mathrm{g} / \mathrm{mL}$ puromycin for 7 days to select the XBP1s overexpressing cells.

\section{Cell Migration and Invasion Assay}

The XBP1s overexpressed cells and control cells were resuspended in FBS-free medium and seeded in the top chamber of transwell plates (Corning-Costar, USA) at a density of $2 \times 10^{4}$ cells/well, with or without Matrigel (1:8 dilution, $50 \mu \mathrm{L}$, BD Biosciences, CA, USA). The bottom chambers were filled with $600 \mu \mathrm{L}$ medium containing $20 \%$ fetal bovine serum. After $24 \mathrm{~h}$, the cells were fixed with $4 \%$ formaldehyde and stained with crystal violet. Unpenetrated cells that remained on the upper surface of chamber were carefully erased from the membrane. The invasive cells on the bottom surface were imaged and counted.

\section{Wound Healing Assay}

The cells were seeded into 6-well-plates. When the cells cover the entire well, we wounded the cells with $200 \mu \mathrm{L}$ sterile pipette tips. After washing off the floating cells with 
PBS, the cells were cultured in FBS-free medium. The photos were taken under the microscope at 0,24 and 48 hours after injury. Then, we use the width of the wound at $0 \mathrm{~h}$ minus the width of $24 \mathrm{~h}$ or $48 \mathrm{~h}$ as the healing distance during that period. And the percentage of this distance to the $0 \mathrm{~h}$ width is the Wound healing rate. Namely: Wound Healing rate $=\left(\right.$ width $\left._{0 \mathrm{~h}}-\mathrm{width}_{\mathrm{t}}\right) / \mathrm{width}_{0 \mathrm{~h}} * 100 \%$.

\section{Western Blot Analysis}

Western Blotting was carried out as described previously. ${ }^{13}$ The following primary antibodies were used: XBP1s (1:250, CST, \#83418), ZO-1 (1:1000, Proteintech, \#21773-1-AP), N-cadherin (1:1000, Proteintech, \#22018-1-AP), E-cadherin (1:1000, Proteintech, \#21773-1-AP), Vimentin (1:1000, Proteintech, \# 20874-1-AP), PAX8 (1:1000, Proteintech, \#10336-1-AP), NIS (1:1000, Proteintech, \#24324-1-AP), andGAPDH (1:1000, Proteintech, \#10494-1-AP).

\section{Immunofluorescence}

Different groups of BCPAP cells were seeded on coverslips and cultured in RPMI 1640 supplied with 10\% FBS overnight. After washing, fixing and permeabilizing, we incubated the coverslips with primary antibodies (antiN-cadherin and anti-E-cadherin, 1:100 dilution, Proteintech) at $4{ }^{\circ} \mathrm{C}$ overnight, respective fluorescence conjugated secondary antibodies were applied thereafter. The slides were imaged using the fluorescence microscope (FV1000; Olympus, Center Valley, PA) under constant laser intensity. Then, we use ImageJ to quantitatively analyze the fluorescence signals from more than three repeated experiments.

\section{Statistical Analysis}

If not mentioned otherwise, data were shown as the mean \pm standard deviation (SD). Difference between two groups was tested using two-tailed Student's $T$-test using GraphPad Prism 8. And Chi square analysis was performed with SPSS 16.0 statistical software (IBM, NY). Statistical significance is considered with $\mathrm{p}$-value $<0.05$.

\section{Results}

\section{Low Expression of XBPIs is Closely Related to Lymph Node Metastasis in PTC}

The clinicopathological characteristics of enrolled PTC patients are summarized in Table 1. Briefly, $87.8 \%$ (36/ 41) of the patients were less than 55 years old, the percentage of patients with lymph node metastasis were $46.3 \%(19 / 41)$ in female and $48.8 \%(21 / 41)$ in male. All the tumors were unifocal, no extrathyroidal extension, and the tumor sizes were between 1 and $2 \mathrm{~cm}$.

The representative images of XBP1s staining in $\mathrm{N} 1$ and $\mathrm{N} 0$ groups were presented in Figure 1A. According to the SI of IHC, we compared the expression of XBP1s in N0 and N1 groups. We observed the expression of XBP1s was significantly decreased in $\mathrm{N} 1$ group as compared to N0 group (Figure 1B). Next, we divided the patients into two groups according to the XBP1s expression level. To identify the optimal cutoff value, we conducted receiver operating characteristic (ROC) curve analyses with SPSS 16.0 statistical software (IBM, NY) to distinguish N1 patients. The cutoff value for XBP1s (area under the curve $[\mathrm{AUC}]=0.81$ ) was 1.05 (Figure $1 \mathrm{C}$ ). The multivariate analysis suggested that $\mathrm{XBP} 1 \mathrm{~s}$ expression was an independent risk factor for lymph node metastasis $(\mathrm{P}=0.003$, hazard ratio $[\mathrm{HR}]=0.066)($ Table 2$)$

\section{Decreased XBPI Expression in PTC Tissue is Associated with Poor Patient Prognosis}

To verify the role of XBP1 in thyroid cancer, we analyzed the transcript levels of XBP1 in non-tumor thyroid and thyroid cancer tissues via UALCAN. ${ }^{14}$ We found that the XBP1's transcript was significantly lower in cancer than in non-tumor thyroids $(\mathrm{P}<0.001$, Figure $2 \mathrm{~A})$. Furthermore, the level of XBP1 transcription was much lower in the patients with lymph node metastasis ( $\mathrm{P}=0.007$, Figure $2 \mathrm{~B}$ ), which was consistent with our previous findings. We then analyzed the original data from TCGA database, and lower expression of XBP1 were reproductively observed in

Table I Clinicopathological Characteristics of Patients with PTC

\begin{tabular}{|c|c|c|}
\hline \multicolumn{2}{|l|}{ Characteristics } & \multirow{2}{*}{$\begin{array}{c}\text { No. of Patients (\%) } \\
4 \mathrm{I}\end{array}$} \\
\hline Patients & & \\
\hline \multirow[t]{2}{*}{ Age (years) } & $<55$ & $36(87.8)$ \\
\hline & $\geq 55$ & $5(12.2)$ \\
\hline \multirow[t]{2}{*}{ Gender } & Male & $22(53.7)$ \\
\hline & Female & $19(46.3)$ \\
\hline Tumor size & $\mathrm{I} \sim 2 \mathrm{~cm}$ & $41(100)$ \\
\hline Tumor focality & Unifocal & $41(100)$ \\
\hline Extrathyroidal extension & No & $41(100)$ \\
\hline \multirow[t]{2}{*}{ Lymph node metastasis } & No & $21(5 \mid .2)$ \\
\hline & $\mathrm{NI}$ & $20(48.8)$ \\
\hline
\end{tabular}


A

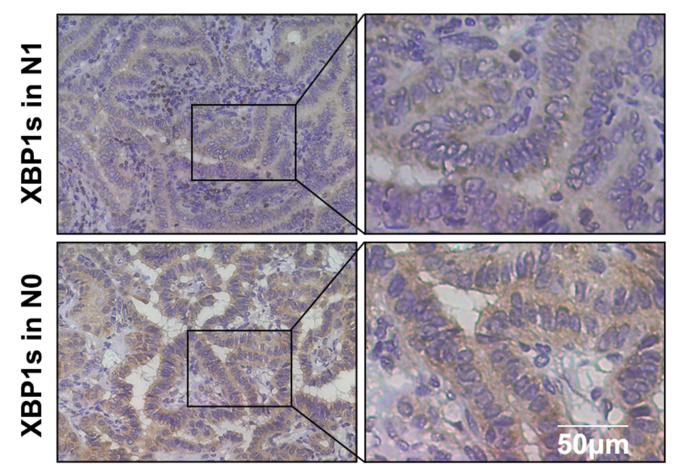

B

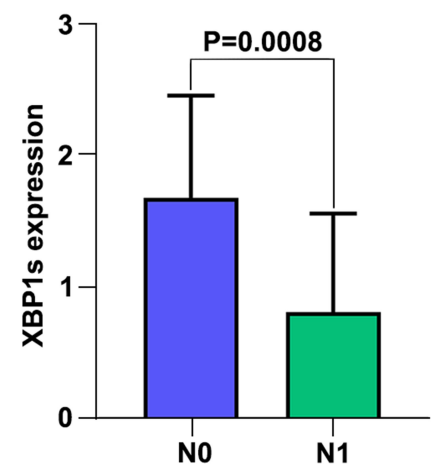

C

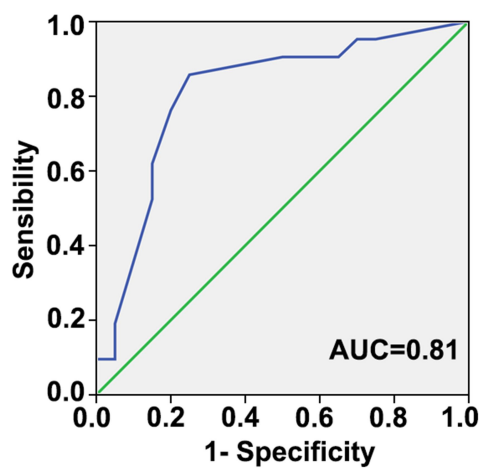

Figure I Low expression of XBPIs is closely related to lymph node metastasis in PTC. (A) Representative immunohistochemical staining profile of PTC tissue samples with (NI) or without (N0) lymph node metastasis. (B) The expression of XBPIs in NI and N0 groups according to the $\mathrm{SI}$ of IHC. (C) ROC curve analyses to distinguish NI patients. Differences between groups in (B) assay were analyzed by two-tailed Student's T-test. Error bars represent means \pm SD.

patients with lymph node metastasis $(\mathrm{P}=0.012$, Figure $2 \mathrm{C})$. Stratification analysis according to the pathological stages indicated that the low XBP1 expression was closely associated with TC stages $(\mathrm{P}<0.05$, Figure 2D). Similar result was obtained $(\mathrm{P}<0.001$, Figure $2 \mathrm{E})$ using the GEPIA dataset. ${ }^{15}$ Moreover, we also found that the high XBP1 expression $[\mathrm{n}($ high $)=255, \mathrm{n}($ low $)=255)]$ was not associated with $\mathrm{OS}(\mathrm{HR}=1, \mathrm{P}=0.99$, Figure $2 \mathrm{~F}$ ) but with good DFS $(\mathrm{HR}=0.47, \mathrm{P}=0.013$, Figure $2 \mathrm{G})$. After normalization, the decreased XBP1 expression was significantly associated with gender, $\mathrm{T}$ classification, lymph node metastasis and stage ( $\mathrm{P}<0.05$, Table 3$)$. Hence, we demonstrated that decreased XBP1 expression was associated with poor TC prognosis.

\section{Overexpression of XBPIs Impairs the Invasive and Metastatic Potential of PTC Cells}

To evaluate whether XBP1s affects migratory and invasive capabilities of PTC cells, we overexpressed XBP1s in

Table 2 Multivariate Survival Analysis of Risk Factors for Lymph Node Metastasis of PTC

\begin{tabular}{|l|c|c|}
\hline \multirow{2}{*}{ Variable } & \multicolumn{2}{|c|}{ Multivariate Analysis } \\
\cline { 2 - 3 } & P-value & HR (95\% Cl) \\
\hline Age (years) & 0.417 & $0.968(0.895 \sim 1.047)$ \\
Gender (female/male) & 0.759 & $1.313(0.231 \sim 7.473)$ \\
T (mm) & 0.234 & $1.214(0.882 \sim 1.669)$ \\
XBPIs expression (low/high) & $\mathbf{0 . 0 0 3}$ & $0.066(0.01 \mathrm{I} \sim 0.392)$ \\
\hline
\end{tabular}

Note: Bold and italic text values indicate $\mathrm{P}<0.05$, which is statistically significant.
BCPAP, KTC-1 and TPC-1 cells using lentivirus transduction, which was then confirmed by immunoblot analysis (Figure 4B). Then, transwell and wound-healing assays were performed. Intriguingly, overexpression of XBP1s significantly suppressed the migration and invasion capacities of BCPAP, KTC-1 and TPC-1 cells (Figure 3A and $\mathrm{B})$.

\section{Overexpression of XBPIs Inhibits EMT in PTC Cells}

To evaluate whether XBP1s regulates EMT, we first explored the correlation between XBP1 and classical EMT markers, including ZO-1, N-cadherin, E-cadherin and Vimentin by analyzing GEPIA. Our results indicated that the XBP1's expression was significantly correlated with ZO-1, $\mathrm{N}$-cadherin, E-cadherin and Vimentin expression (Figure 4A). Then, we evaluated the EMT process in XBP1s overexpressed BCPAP, KTC-1 and TPC-1 cells. We found significantly increased ZO-1 and E-cadherin expression and decreased N-cadherin expression in XBP1s overexpressed cells, and no obvious difference of Vimentin expression between XBP1s overexpressed and control cells (Figure 4B). Similarly, we observed upregulated E-cadherin and downregulated N-cadherin in BCPAP cells when XBP1s was overexpressed as determined by immunofluorescence (Figure 4C). These data collaboratively suggested that XBP1s inhibits EMT of PTC cells.

\section{Discussion}

Although the majority of PTC patients have relatively good prognosis, some patients might experience cancer recurrence 

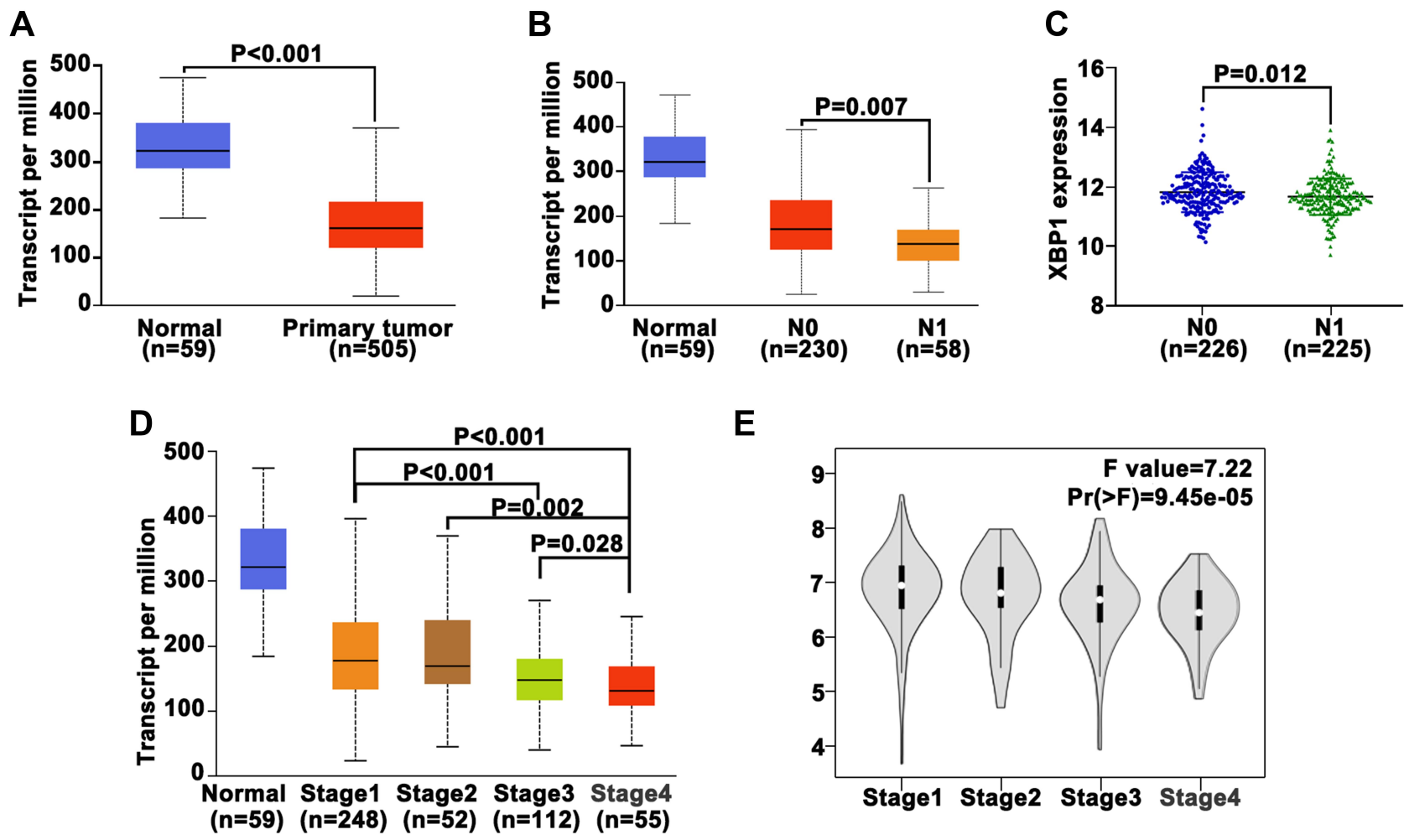

E

F

G
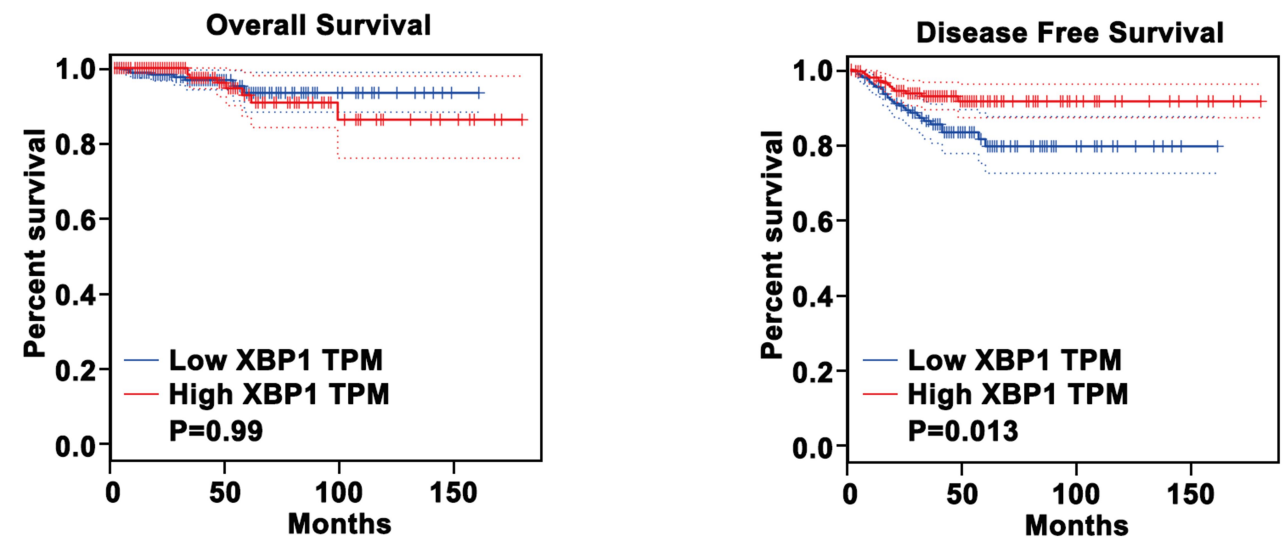

Figure 2 Decreased XBPI expression in PTC tissue is associated with patient prognosis. (A-C) Relative levels of XBPI transcripts in 505 TC, 230 TC whit N0, 58 TC with $\mathrm{NI}$ and 59 non-tumor thyroid tissues in TCGA from UALCAN and original data. (D) (UALCAN), (E) (GEPIA) stratification analysis of XBPI expression in the different stages of TC. (F and $\mathbf{G})$ Kaplan-Meier curves showing the relationship of XBPI expression to OS and DFS of patients with TC.

or/and metastasize to the cervical lymph nodes as well as distant organs. It can severely impair the survival and life quality. Thus, identifying molecular markers for metastasis and understanding their pathological functions is utmost important to improve PTC patients with metastasis.

$\mathrm{XBP} 1$ is a key regulator of UPR, which has been proved to be an oncogenic driver in many cancers, such as prostate cancer, ${ }^{9}$ breast cancer, ${ }^{10}$ hepatocellular carcinoma, ${ }^{16}$ pulmonary adenocarcinoma, ${ }^{17}$ oral squamous cell carcinoma. ${ }^{18}$ However, our data suggested that XBP1 played a tumor suppressor role in PTC, which was not only supported by publicly accessible dataset but also our analysis with inhouse PTC patients' samples as well as in vitro PTC cell lines. We showed that the XBP1 transcription was significantly decreased in TC tissues as compared to non-tumor thyroid tissues. Furthermore, XBP1s expression was much lower in PTC patients with cervical lymph node metastasis than nonmetastasis patients. Moreover, we also found that XBP1 expression was significantly correlated with patients' gender, T classification, lymph node metastasis and PTC stages. And 
Table 3 Association of XBPI Expression with Clinicopathologic Features in PTC Patients in TCGA

\begin{tabular}{|c|c|c|c|c|c|}
\hline \multirow[t]{2}{*}{ Parameter } & \multirow[t]{2}{*}{ Total $n=45 I$ (\%) } & \multicolumn{2}{|c|}{ XBPI Expression } & \multirow[t]{2}{*}{$\chi^{2}$} & \multirow[t]{2}{*}{$\mathbf{P}$} \\
\hline & & Low $n=235$ (\%) & High n=216 (\%) & & \\
\hline Age (years) & & & & 2.418 & 0.120 \\
\hline$<55$ & $299(66.3)$ & $148(49.5)$ & $|5|(50.5)$ & & \\
\hline$\geq 55$ & $152(33.7)$ & $87(57.2)$ & $65(42.8)$ & & \\
\hline Gender & & & & 4.810 & 0.028 \\
\hline Female & $327(72.5)$ & $160(48.9)$ & $167(51.1)$ & & \\
\hline Male & $124(27.5)$ & $75(60.5)$ & $49(39.5)$ & & \\
\hline Tumor size & & & & 2.095 & 0.148 \\
\hline$\leq 2 \mathrm{~cm}$ & $164(36.4)$ & 79 (48.2) & 85 (5I.8) & & \\
\hline$>2 \mathrm{~cm}$ & $273(60.5)$ & I5I (55.3) & $122(44.7)$ & & \\
\hline Tumor focality & & & & 0.053 & 0.817 \\
\hline Unifocal & $183(40.6)$ & $102(55.7)$ & $81(44.3)$ & & \\
\hline Multifocal & 135 (29.9) & $77(57.0)$ & $58(43.0)$ & & \\
\hline T classification & & & & 13.369 & 0.000 \\
\hline TI-T2 & $272(60.3)$ & $|2|(44.5)$ & I5I (55.5) & & \\
\hline T3-T4 & $170(37.7)$ & $106(62.4)$ & $64(37.6)$ & & \\
\hline Lymph node metastasis & & & & 9.984 & 0.002 \\
\hline No & $226(50.1)$ & I0I (44.7) & $125(55.3)$ & & \\
\hline $\mathrm{NI}$ & $225(49.9)$ & $134(59.6)$ & $91(40.4)$ & & \\
\hline Metastasis & & & & 0.636 & 0.425 \\
\hline Mo & $270(59.9)$ & $152(56.3)$ & $118(43.7)$ & & \\
\hline MI & $7(1.6)$ & $5(7 \mid .4)$ & $2(28.6)$ & & \\
\hline Stage & & & & 18.234 & 0.000 \\
\hline I-II & $294(65.2)$ & I 32 (44.9) & $162(55.1)$ & & \\
\hline III-IV & $156(34.6)$ & $103(66.0)$ & $53(34.0)$ & & \\
\hline Histological type (TPC) & & & & 5.205 & 0.074 \\
\hline Classical/usual & $332(73.6)$ & I8I (54.5) & $|5|(45.5)$ & & \\
\hline Follicular & $78(17.3)$ & $32(41.0)$ & $46(59.0)$ & & \\
\hline Tall cell & $34(7.5)$ & $20(58.8)$ & $14(4 \mid .2)$ & & \\
\hline
\end{tabular}

Note: Bold and italic text values indicate $P<0.05$, which is statistically significant.

low XBP1 expression was associated with poor DFS but not with overall survival, which is really interesting. Due to the good prognosis of most PTC clinically, the difference in OS is usually not observed in PTC patients, but DFS is closely related to lymph node metastasis. Therefore, the results of the survival analysis coincide with the actual clinical situation and provide a strong evidence that XBP1s can be used as PTC lymph node metastasis. These data collectively suggest that the $\mathrm{XBP} 1 \mathrm{~s}$ expression can serve as a prognostic factor for PTC.

In order to verify our conclusion obtained from the clinical samples, we carried out molecular studies with PTC cell lines. We found that XBP1 overexpression inhibited the PTC cells' invasion, migration, and wound healing capacity. Mechanistically, XBP1s could enhance the expression of classical EMT markers such as ZO-1 and E-cadherins, which might contribute to suppression of invasion and migration of PTC cells. These results further indicated that XBP1s might acted as a PTC suppressor, which was in line with the observations from the clinical samples.

Previous studies have shown that activation of XBP1s leads to expansion of the endoplasmic reticulum (ER). And it is pivotal for cellular differentiation in various secretory cell types. ${ }^{19-22}$ XBP1 is essential for plasma cell differentiation and secretory organ development. ${ }^{21,23}$ Mist1 is required for maintenance of some secretory cell 
A
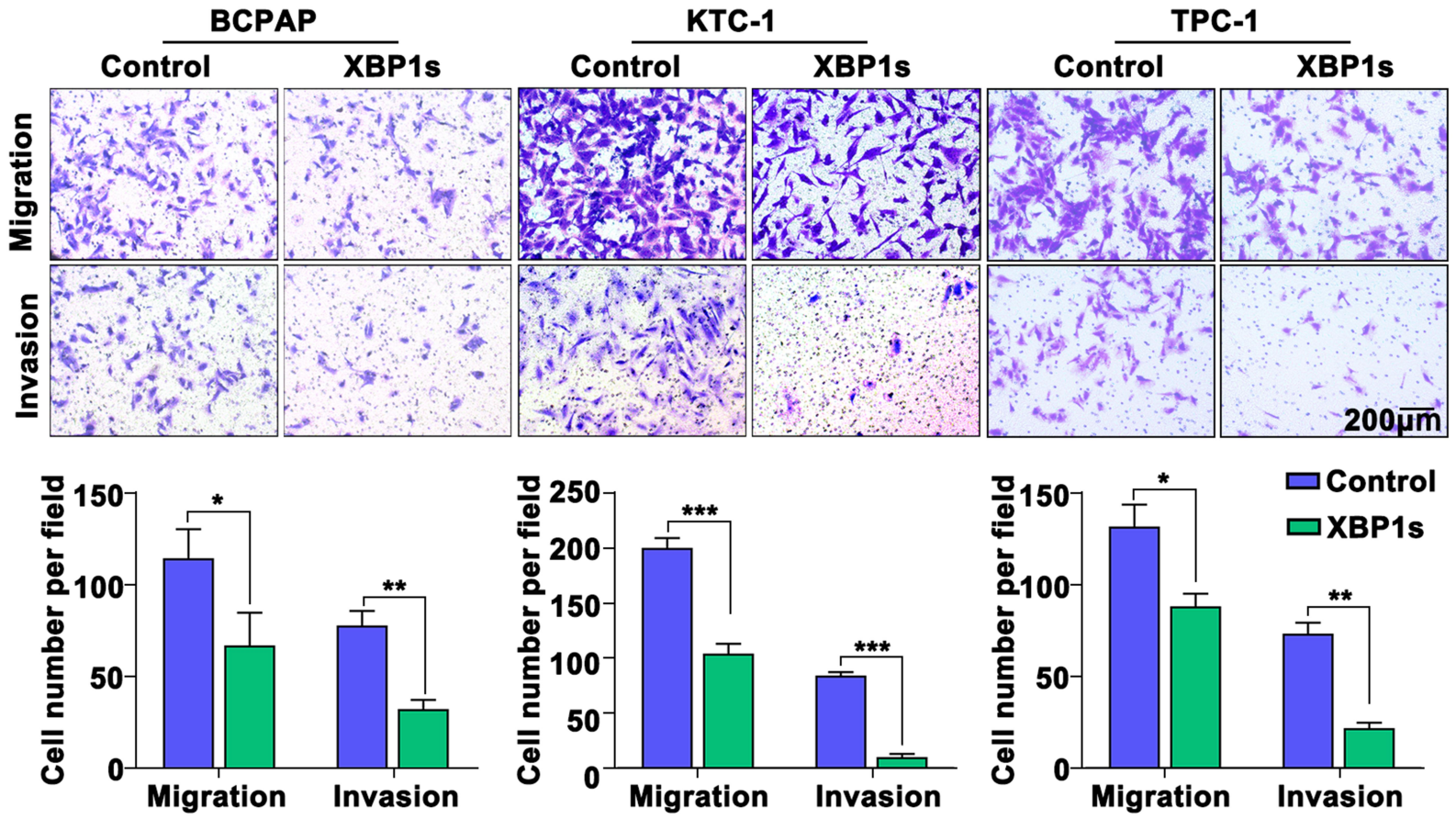

B
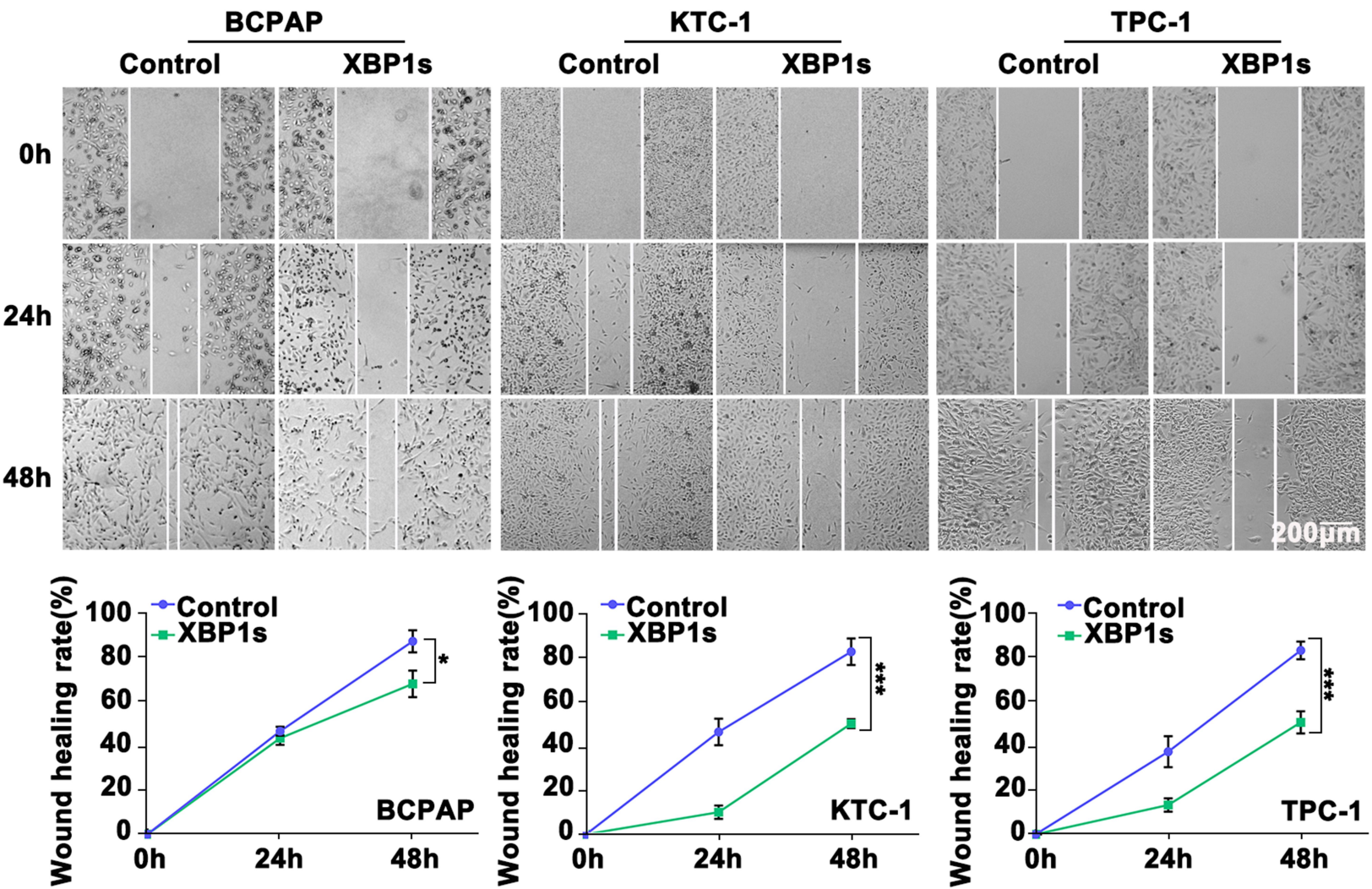

Figure 3 Overexpression of XBPIs reduces the invasive and metastatic potential of PTC cells, and inhibits EMT in PTC cells. (A) Cell migration and invasion assay. (B) Wound healing assay. Difference between two groups was tested using two-tailed Student's $T$-test. Data are expressed as the mean \pm SD of each group of cells from three separate experiments. ${ }^{*} \mathrm{P}<0.05 ; * * \mathrm{P}<0.0 \mathrm{I} ; * * * \mathrm{P}<0.00 \mathrm{I}$. 

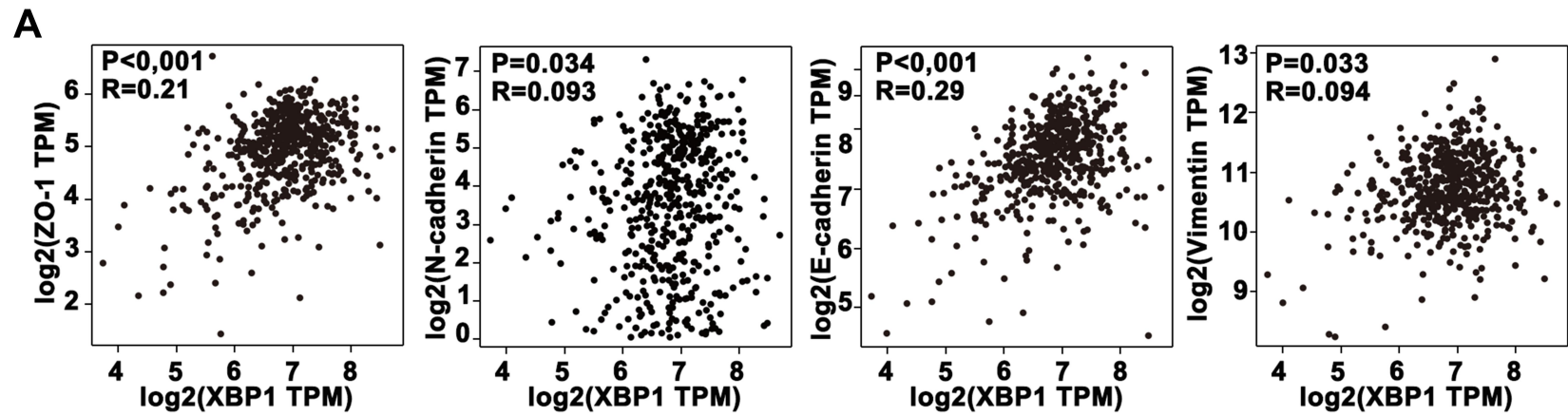

B
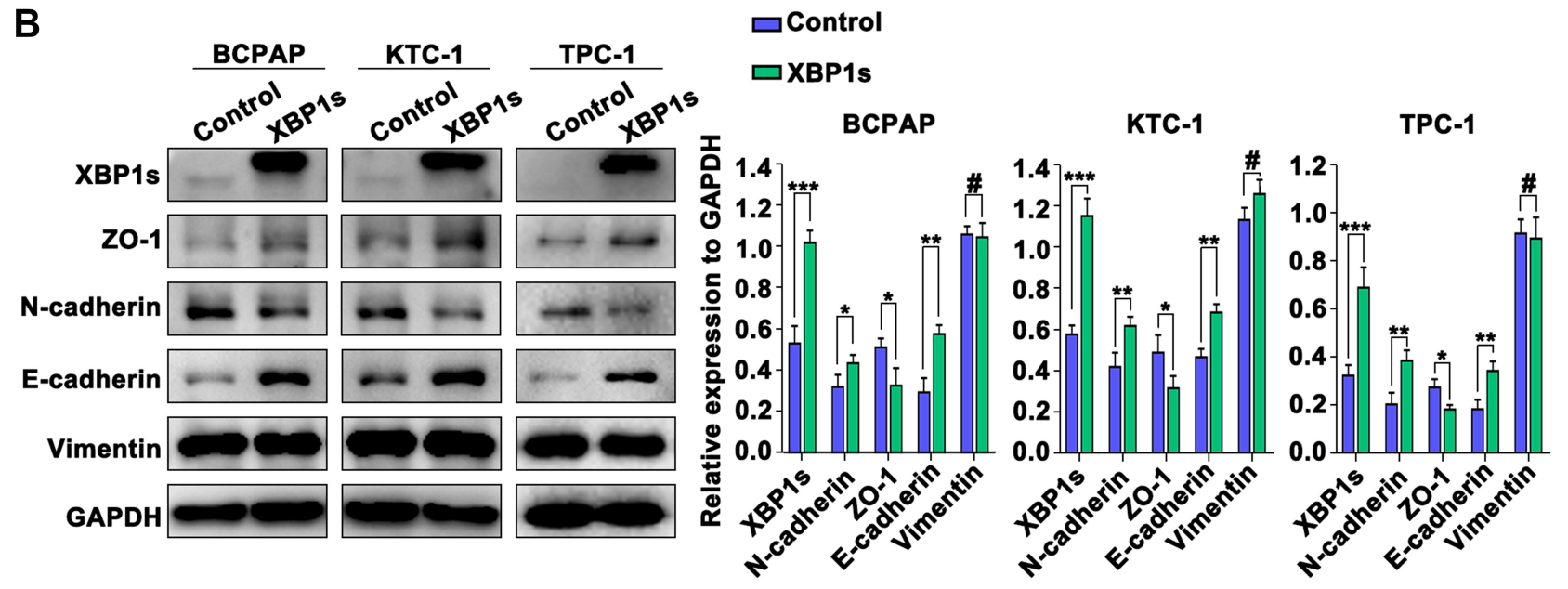

KTC-1

TPC-1

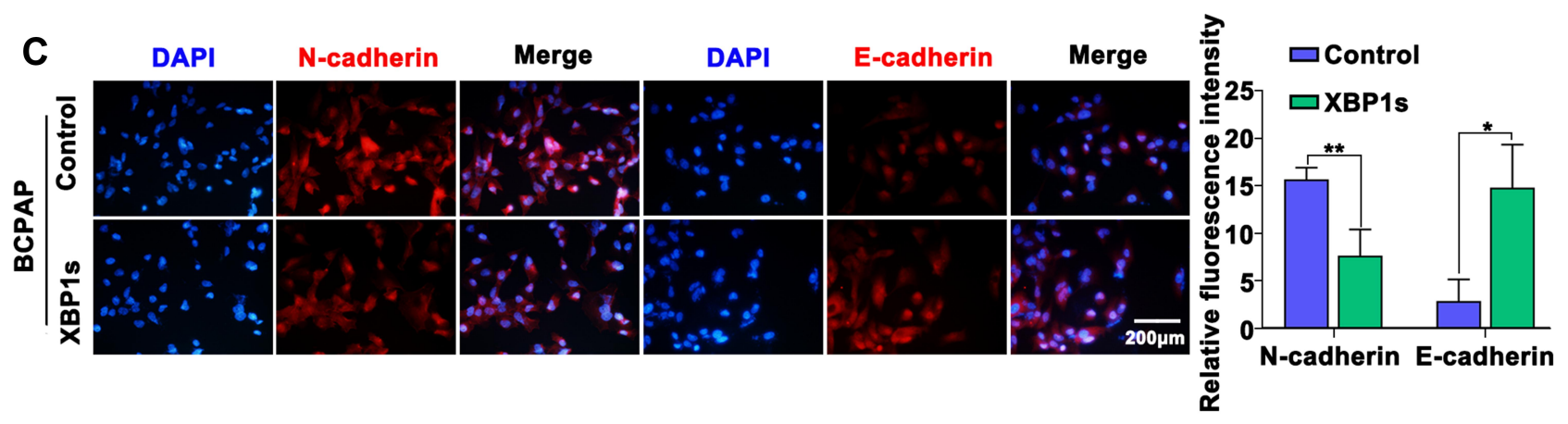

Figure 4 Overexpression of XBPIs inhibits EMT in PTC cells. (A) Correlation analysis between XBPI and EMT-related markers (ZO-I, N-cadherin, E-cadherin and Vimentin) in TCGA (GEPIA). (B) Western blot analysis of the expression of XBPIs and EMT-related markers in PTC cells. (C) Immunofluorescence analysis of the expression of $\mathrm{N}$-cadherin and E-cadherin in PTC cells. Difference between two groups was tested using two-tailed Student's T-test. Data are expressed as the mean \pm SD of each group of cells from three separate experiments. ${ }^{*} \mathrm{P}<0.05 ; * * \mathrm{P}<0.01$; ${ }^{*} * * \mathrm{P}<0.00 \mathrm{I},{ }^{*} \mathrm{P}>0.05$.

identity and maturation. And it was reported to be a direct target of XBP1.22 Thyroid cancer is a tumor derived from endocrine system, which can secrete thyroid hormone and thyroglobulin. This special characteristic of thyroid carcinoma may provide a specific context for XBP1s to play different roles between thyroid carcinoma and the other tumors. Indeed, the expression of XBP1 and Mist1 are closely related in thyroid carcinoma samples (Supp. Figure 1A). Therefore, we speculate that XBP1s may regulate the differentiation of thyroid follicular cells, and we will investigate and validate this conjecture in future work.

By accumulating mutations, thyroid cancer cells constantly undergo de-differentiating, which can cause tumorigenesis and tumor progression from well-differentiated carcinomas to poorly differentiated and even to undifferentiated carcinomas. ${ }^{24}$ Therefore, we speculate that XBP1s may be a robust molecular marker to determine the differentiation of thyroid carcinoma. Then, we performed Western blot experiments to detect the expression of 
PAX8 and NIS, which are thyroid-specific markers, in thyroid cancer after overexpression of XBP1s. We found that both PAX8 and NIS were up-regulated when XBP1s was overexpressed (Supp. Figure 1B), which is consistent with our conjecture. Poorly differentiated and undifferentiated tumors are more malignant and losing their secretory function in accordance with XBP1s suppression. It was reported that enforced expression of XBP1s resulted in reduction of stemness and proliferation of colorectal cancer cells, as activation of XBP1s was capable of activating PERK-eIF2 $\alpha$ signaling, which might reduce the global protein synthesis rates and induced apoptosis in these cells. ${ }^{12}$ Whether XBP1s has a similar molecular function in thyroid cancer requires future investigation.

\section{Conclusion}

In summary, our data indicate that the expression of $\mathrm{XBP} 1 \mathrm{~s}$ is down-regulated in thyroid cancer tissues and associated with $\mathrm{T}$ classification, lymph node metastasis and PTC stages. Functionally, XBP1s acts as a tumor suppressor to inhibit the EMT process and metastasis of PTC cells. These findings suggest that XBP1s expression is a prognostic factor for thyroid carcinoma patients, and sustaining XBP1s expression might be a new strategy to control PTC progression.

\section{Ethical Statement}

Written informed consent was obtained from every PTC patient whose clinical specimens were used for the current research. The study was approved by the Ethics Committee of Tongji Medical College, Huazhong University of Science and Technology, China.

\section{Acknowledgments}

This work was supported by a grant from the National Natural Science Foundations of China (No. 81672611). The authors gratefully acknowledge Dr. Zili Zhou for his kind advice and guidance throughout the experiments.

\section{Disclosure}

The authors report no conflicts of interest in this work.

\section{References}

1. Rosenbaum MA, McHenry CR. Contemporary management of papillary carcinoma of the thyroid gland. Expert Rev Anticancer Ther. 2009;9(3):317-329. doi:10.1586/14737140.9.3.317

2. Brito JP, Hay ID, Morris JC. Low risk papillary thyroid cancer. $B M J$. 2014;348:g3045. doi:10.1136/bmj.g3045
3. Adam MA, Pura J, Goffredo P, et al. Presence and number of lymph node metastases are associated with compromised survival for patients younger than age 45 years with papillary thyroid cancer. $J$ Clin Oncol. 2015;33(21):2370-2375. doi:10.1200/ JCO.2014.59.8391

4. Walter P, Ron D. The unfolded protein response: from stress pathway to homeostatic regulation. Science. 2011;334(6059):1081-1086. doi:10.1126/science. 1209038

5. Hotamisligil GS. Endoplasmic reticulum stress and the inflammatory basis of metabolic disease. Cell. 2010;140(6):900-917. doi:10.1016/j. cell.2010.02.034

6. Clarke HJ, Chambers JE, Liniker E, et al. Endoplasmic reticulum stress in malignancy. Cancer Cell. 2014;25(5):563-573. doi:10.1016/ j.ccr.2014.03.015

7. Wang M, Kaufman RJ. The impact of the endoplasmic reticulum protein-folding environment on cancer development. Nat Rev Cancer. 2014;14(9):581-597. doi:10.1038/nrc3800

8. Chevet E, Hetz C, Samali A. Endoplasmic reticulum stress-activated cell reprogramming in oncogenesis. Cancer Discov. 2015;5 (6):586-597. doi:10.1158/2159-8290.CD-14-1490

9. Sheng X, Nenseth HZ, Qu S, et al. IRE1alpha-XBP1s pathway promotes prostate cancer by activating c-MYC signaling. Nat Commun. 2019;10(1):323. doi:10.1038/s41467-018-08152-3

10. Chen X, Iliopoulos D, Zhang Q, et al. XBP1 promotes triple-negative breast cancer by controlling the HIF1alpha pathway. Nature. 2014;508(7494):103-107. doi:10.1038/nature13119

11. Niederreiter L, Fritz TM, Adolph TE, et al. ER stress transcription factor Xbp1 suppresses intestinal tumorigenesis and directs intestinal stem cells. J Exp Med. 2013;210(10):2041-2056. doi:10.1084/ jem. 20122341

12. Spaan CN, Smit WL, van Lidth DJJ, et al. Expression of UPR effector proteins ATF6 and XBP1 reduce colorectal cancer cell proliferation and stemness by activating PERK signaling. Cell Death Dis. 2019;10(7):490. doi:10.1038/s41419-019-1729-4

13. Yang W, Zheng Z, Yi P, et al. LncRNA NBR2 inhibits the malignancy of thyroid cancer, associated with enhancing the AMPK signaling. Front Oncol. 2020;10:956. doi:10.3389/fonc.2020.00956

14. Chandrashekar DS, Bashel B, Balasubramanya S, et al. UALCAN: a portal for facilitating tumor subgroup gene expression and survival analyses. Neoplasia. 2017;19(8):649-658. doi:10.1016/j. neo.2017.05.002

15. Tang Z, Li C, Kang B, et al. GEPIA: a web server for cancer and normal gene expression profiling and interactive analyses. Nucleic Acids Res. 2017;45(W1):W98-W102. doi:10.1093/nar/gkx247

16. Wu S, Du R, Gao C, et al. The role of XBP1s in the metastasis and prognosis of hepatocellular carcinoma. Biochem Biophys Res Commun. 2018;500(3):530-537. doi:10.1016/j.bbrc.2018.04.033

17. Kwon D, Koh J, Kim S, et al. Overexpression of endoplasmic reticulum stress-related proteins, XBP1s and GRP78, predicts poor prognosis in pulmonary adenocarcinoma. Lung Cancer. 2018;122:131-137. doi:10.1016/j.lungcan.2018.06.005

18. Sun Y, Jiang F, Pan Y, et al. XBP1 promotes tumor invasion and is associated with poor prognosis in oral squamous cell carcinoma. Oncol Rep. 2018;40(2):988-998. doi:10.3892/or.2018.6498

19. Sriburi R, Jackowski S, Mori K, et al. XBP1: a link between the unfolded protein response, lipid biosynthesis, and biogenesis of the endoplasmic reticulum. J Cell Biol. 2004;167(1):35-41. doi:10.1083/ jcb.200406136

20. Back SH, Scheuner D, Han J, et al. Translation attenuation through eIF2alpha phosphorylation prevents oxidative stress and maintains the differentiated state in beta cells. Cell Metab. 2009;10(1):13-26. doi:10.1016/j.cmet.2009.06.002

21. Reimold AM, Iwakoshi NN, Manis J, et al. Plasma cell differentiation requires the transcription factor XBP-1. Nature. 2001;412 (6844):300-307. doi:10.1038/35085509 
22. Acosta-Alvear D, Zhou Y, Blais A, et al. XBP1 controls diverse cell type- and condition-specific transcriptional regulatory networks. $\mathrm{Mol}$ Cell. 2007;27(1):53-66. doi:10.1016/j.molcel.2007.06.011

23. Lee AH, Chu GC, Iwakoshi NN, et al. XBP-1 is required for biogenesis of cellular secretory machinery of exocrine glands. EMBO J. 2005;24(24):4368-4380. doi:10.1038/sj.emboj.7600903
24. Haugen BR, Sherman SI. Evolving approaches to patients with advanced differentiated thyroid cancer. Endocr Rev. 2013;34 (3):439-455. doi:10.1210/er.2012-1038

\section{Publish your work in this journal}

OncoTargets and Therapy is an international, peer-reviewed, open access journal focusing on the pathological basis of all cancers, potential targets for therapy and treatment protocols employed to improve the management of cancer patients. The journal also focuses on the impact of management programs and new therapeutic

Submit your manuscript here: https://www.dovepress.com/oncotargets-and-therapy-journal agents and protocols on patient perspectives such as quality of life, adherence and satisfaction. The manuscript management system is completely online and includes a very quick and fair peer-review system, which is all easy to use. Visit http://www.dovepress.com/ testimonials.php to read real quotes from published authors. 